\title{
OPEN Longitudinal analysis of premotor anthropometric and serological markers of Parkinson's disease
}

\author{
Katsunori Yokoi ${ }^{1}$, Makoto Hattori ${ }^{1}$, Yuki Satake ${ }^{1}$, Yasuhiro Tanakaa ${ }^{1}$, Maki Sato ${ }^{1}$, \\ Atsushi Hashizume ${ }^{1}$, Akihiro Hori' ${ }^{2}$, Motoshi Kawashima ${ }^{3}$, Akihiro Hirakawa ${ }^{4}$, \\ Hirohisa Watanabe ${ }^{5,6} \&$ Masahisa Katsuno ${ }^{1 \bowtie}$
}

Parkinson's disease (PD) is a debilitating neurodegenerative disorder in which nonmotor symptoms, such as constipation and hyposmia, precede the onset of motor symptoms by 20 years. The aim of this study was to identify biomarkers at the premotor stage of PD. We assessed the differences in longitudinal changes in anthropometric and serological indices obtained from health check-up data before and after the onset of motor symptoms between male and female PD patients and healthy subjects. We enrolled 22 male and 23 female PD patients and 60 male and 60 female healthy controls. A mixed-effects model was used to estimate the trajectory of each clinical marker over the years before and after motor symptoms onset in the PD subjects, which were then compared with the trajectories of the healthy controls. The results showed a premotor blood pressure increase in female PD patients and premotor decreases in haematocrit, total cholesterol and low-density lipoprotein cholesterol in the male patients. Our results indicated that blood pressure, haematocrit and serum cholesterol levels are potential premotor markers of PD. Additionally, the changes in anthropometric and serological indices before PD motor symptoms onset were sex specific.

Parkinson's disease (PD) is the second most common neurodegenerative disorder after Alzheimer's disease. The frequency of the disorder is relatively high at 1.3 cases per 10,0000 people $<45$ years old, 3100 per 10,0000 in those $75-85$ years old and 4300 per 10,0000 in those $>85$ years old ${ }^{1,2}$. PD is characterised by motor signs of bradykinesia, rigidity and resting tremor, and by nonmotor symptoms, including cognitive, neuropsychiatric, sleep, autonomic and sensory disturbances ${ }^{3,4}$. Some nonmotor symptoms, such as constipation, rapid eye movement sleep behaviour disorder (RBD), hyposmia and depression, are known as premotor/prodromal symptoms ${ }^{5,6}$. Because these symptoms manifest decades before motor symptoms, the pathogenesis of PD is thought to emerge long before the onset of motor symptoms. This view is supported by the histopathological observation that $>50 \%$ of dopaminergic neurons are lost at the onset of motor symptoms ${ }^{7}$.

In neurodegenerative diseases, including PD, attempts to identify biological markers for the preclinical stages have received increased attention because several studies have identified potential preclinical biomarkers. For instance, serum neurofilament light-chain levels are elevated in the preclinical stage of Alzheimer's disease (AD) and amyotrophic lateral sclerosis/frontotemporal lobar degeneration ${ }^{8-10}$. The cerebrospinal fluid levels of amyloid beta and tau are established preclinical biomarkers for $\mathrm{AD}^{11}$. Dipeptide repeat protein is another potential preclinical marker for C9ORF72-associated amyotrophic lateral sclerosis ${ }^{12}$. Neuroimaging and neurophysiological alteration have also been detected in the preclinical phase of spinocerebellar ataxias and Huntington's disease ${ }^{13,14}$.

The mean height of young adults is significantly lower in those affected by PD than in controls ${ }^{15}$. Weight loss in PD patients has appeared to be a continuous process that starts several years before the diagnosis of $\mathrm{PD}^{16}$. Anaemia is associated with PD and may precede the onset of motor symptoms by $\geq 20$ years ${ }^{17}$. Higher serum levels of total and low-density lipoprotein (LDL) cholesterol (T-Cho and LDL-Cho, respectively) have been shown to be associated with a decreased risk of $\mathrm{PD}^{18,19}$, and higher serum levels of urate were also associated with a decreased risk of developing $\mathrm{PD}^{20,21}$.

\footnotetext{
${ }^{1}$ Department of Neurology, Nagoya University Graduate School of Medicine, 65 Tsurumai-cho, Showa-ku, Nagoya 466-8550, Japan. ${ }^{2}$ Kumiai Kosei Hospital, Takayama, Gifu 5068502, Japan. ${ }^{3}$ Medical Examination Centre, Daido Clinic, Nagoya 4578511, Japan. ${ }^{4}$ Department of Clinical Biostatistics, Graduate School of Medical and Dental Sciences, Tokyo Medical and Dental University, Tokyo 1138510, Japan. ${ }^{5}$ Brain \& Mind Research Centre, Nagoya University Graduate School of Medicine, Nagoya 4668560, Japan. ${ }^{6}$ Department of Neurology, Fujita Medical University, Toyoake, Aichi 4701192, Japan. ${ }^{\bowtie}$ email: ka2no@med.nagoya-u.ac.jp
} 
Patients with PD

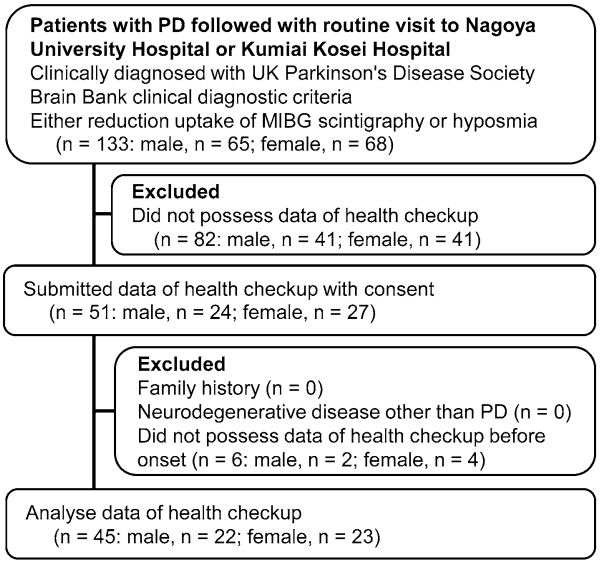

Healthy controls

\begin{tabular}{l}
$\begin{array}{l}\text { Healthy subjects followed with periodic health checkup } \\
\text { at Daido Hospital } \\
\text { Submitted data of health checkup more than } 4 \text { years from } \\
\text { last evaluation with consent } \\
\text { Matched for age and sex with PD patients } \\
(n=187: \text { male, } n=98 ; \text { female, } n=89)\end{array}$ \\
$\qquad \begin{array}{l}\text { Excluded } \\
\text { Parkinson's disease }(n=0) \\
\text { Neurodegenerative disease other than PD }(n=0) \\
\text { Family history of PD } \\
(n=6: \text { male, } n=2 ; \text { female, } n=4) \\
\text { Serious complications } \\
(n=7: \text { male, } n=4 ; \text { female, } n=3) \\
\text { Prodromal symptoms of PD } \\
(n=54: \text { male, } n=32 ; \text { female, } n=22)\end{array}$ \\
$\begin{array}{l}\text { Analyse data of health checkup } \\
(n=120: \text { male, } n=60 ; \text { female, } n=60)\end{array}$ \\
\hline
\end{tabular}

Figure 1. Flowchart of the study population. $\mathrm{PD}=$ Parkinson's disease, $\mathrm{MIBG}=$ metaiodobenzylguanidine.

However, little is known about the longitudinal changes in biomarkers at a premotor stage of PD. Recently, by analysing patients' health check-up longitudinal data, we found that serum creatinine $(\mathrm{Cr})$ levels started to decrease before the onset of neurological symptoms in spinal and bulbar muscular atrophy, a hereditary neuromuscular disease ${ }^{22}$. The aim of the present study was to examine longitudinal changes in biochemical and anthropometric indices at the premotor stage of PD. To this end, we investigated changes before and after motor symptoms onset in PD patients by analysing health check-up longitudinal data.

\section{Results}

Participant characteristics. We recruited a total of 133 patients with PD and 187 age- and sex-matched healthy controls between October 2016 and January 2019 (Fig. 1). We excluded 88 PD patients for whom health check-up data before the onset of motor symptoms were not available; accordingly, we analysed 45 PD patients ( 22 males and 23 females). All included patients submitted their health checkup data $>4$ years from their last evaluation and provided written informed consent. Among the potential healthy controls, we excluded six subjects who had a family history of PD, seven subjects who had serious complications (active cancer, severe anaemia, pancreatitis, epicarditis, inflammatory bowel disease, gastric ulcer or stroke) and 54 individuals who had prodromal symptoms of PD (any of hyposmia, RBD and constipation). Finally, we analysed 120 healthy controls, 60 males and 60 females. There were no significant differences in the mean ages at PD onset between the males and females (Supplemental Fig. 1). The evaluation period in all patients with PD included their premotor period. The profiles of medication history were similar between the PD subjects and healthy controls for males and females (Table 1).

Comparison of baseline data. First, we compared the baseline values of each item between the PD and healthy subjects for each sex separately. We defined the baseline as the onset of motor symptoms for PD groups and as the last evaluation for the healthy subjects. In males, there were significant differences between the PD patients and healthy controls in weight, body mass index (BMI), haematocrit (Ht), T-Cho, LDL-Cho and creatinine $(\mathrm{Cr})$, and all the values of all these indices were lower in male PD patients than in the male healthy controls. In females, significant differences were found in height, systolic blood pressure (BP), diastolic BP, aspartate aminotransferase (AST) and T-Cho between the groups. The values of AST, systolic BP and diastolic BP were higher in female PD patients, whereas the values of other indices were lower in female PD patients than in the female healthy controls (Table 2). There were no significant differences in the clinical backgrounds, such as disease severity and medication, between the male and female patients with PD (Supplemental Table 1).

Longitudinal changes in anthropometric markers. To detect premotor changes in indices, we analysed the estimated trajectories of the factors that showed statistically significant differences at baseline between healthy subjects and those with PD for either sex: height, weight, BMI, systolic BP and diastolic BP (Fig. 2a-e). There was no premotor change in height in either sex (Fig. 2a). The value of weight and BMI decreased after the onset of motor symptoms, but premotor alteration was not observed in both sexes (Fig. 2b,c). By contrast, the values of systolic and diastolic BP were elevated before motor onset in the female PD patients when compared with the female controls, and the difference decreased as the manifestation of motor symptoms increased, although these trends were not detected in males (Fig. 2d,e). To confirm the results of our linear mixed model analysis, we also directly compared the actual data of each item between the PD and healthy subjects for each PD disease stage (Fig. 2f,g). Increases in systolic and diastolic BP were observed $>6$ years before motor onset in the female PD patients, but the differences from the control values decreased with disease progression, as shown in the estimated trajectories. 


\begin{tabular}{|c|c|c|c|}
\hline & Male patients with PD $(n=22)$ & Male healthy controls $(n=60)$ & $p$ value \\
\hline Age at motor symptoms onset (PD patients), y & $65.5 \pm 8.9(48-81)$ & NA & \multirow{2}{*}{0.393} \\
\hline Age at final evaluation (healthy controls), y & NA & $64.8 \pm 7.8(47-81)$ & \\
\hline Total evaluation period, $\mathrm{y}$ & $13.5 \pm 6.4(5-31)$ & $5.7 \pm 0.5(4-7)$ & \\
\hline Initial evaluation from onset, $y$ & $7.9 \pm 5.1(2-20)$ & NA & \\
\hline Last evaluation from onset, $y$ & $4.6 \pm 3.5(0-13)$ & NA & \\
\hline \multicolumn{4}{|l|}{ Medications, n (\%) } \\
\hline Antihypertensive drug & $3(13.6)$ & $14(23.3)$ & 0.539 \\
\hline Antidiabetic drug & $3(13.6)$ & $1(1.7)$ & 0.057 \\
\hline Anticholesterolemic drug & $3(13.6)$ & $5(8.3)$ & 0.437 \\
\hline \multirow[t]{2}{*}{ Antihyperuricemia drug } & $0(0)$ & $1(1.7)$ & 1.000 \\
\hline & Female patients with PD $(n=23)$ & Female healthy controls $(n=60)$ & $p$ value \\
\hline Age at motor symptoms onset (PD patients), y & $67.3 \pm 7.2(51-80)$ & NA & \multirow{2}{*}{0.113} \\
\hline Age at the final evaluation (healthy controls), $y$ & NA & $62.8 \pm 7.5(51-74)$ & \\
\hline Total evaluation period, $\mathrm{y}$ & $10.8 \pm 5.1(4-21)$ & $6.1 \pm 1.1(4-9)$ & \\
\hline Initial evaluation from onset, $y$ & $6.9 \pm 4.8(1-17)$ & NA & \\
\hline Last evaluation from onset, $\mathrm{y}$ & $2.9 \pm 3.5(0-10)$ & NA & \\
\hline \multicolumn{4}{|l|}{ Medications, n (\%) } \\
\hline Antihypertensive drug & $5(21.7)$ & $6(10.0)$ & 0.168 \\
\hline Antidiabetic drug & $0(0)$ & $1(1.7)$ & 1.000 \\
\hline Anticholesterolemic drug & $4(17.4)$ & $3(5)$ & 0.089 \\
\hline Antihyperuricemia drug & $0(0)$ & $0(0)$ & 1.000 \\
\hline
\end{tabular}

Table 1. Clinical backgrounds of the subjects. Data represent the mean \pm standard deviation. PD Parkinson's disease, $N A$ not available.

Longitudinal changes in serological markers. Next, we analysed the estimated trajectories of the blood examination items, which showed statistically significant differences at baseline between PD and healthy subjects for either sex: Ht, AST, T-Cho, LDL-Cho and Cr (Fig. 3a-e). In the male PD patients, the haematocrit values started to decrease before the onset of motor symptoms (Fig. 3a). The serum values of AST increased after onset in the female PD patients, but a premotor change in this factor was not observed in either sex (Fig. 3b). The T-Cho and LDL-Cho values began to decrease before the onset of motor symptoms in the male PD patients (Fig. 3c,d). These values showed progressive declines after the onset in both sexes, but a premotor change was not observed in the female PD patients. There was no premotor change in serum Cr levels in the PD patients in either sex (Fig. 3e). In the direct comparison of actual data, the haematocrit levels in the male PD patients showed a decrease from 5 years to 1 year before the motor onset (Fig. 3f). Serum AST levels in the female PD subjects showed an increase from 10 years to 1 year before the onset of motor symptoms (Fig. $3 \mathrm{~g}$ ). Serum T-Cho levels in the male PD subjects began to decrease 5 to 1 years before the onset of motor symptoms, whereas the values decreased only after motor onset in females (Fig. $3 \mathrm{~h}$ ). Similar results were obtained for the serum LDLCho levels (Fig. 3i).

To exclude the influence of medications (antihypertensive agents and statins) on the longitudinal change in BP and serum lipids, we also performed subgroup analysis on the subjects not taking such drugs. In the subgroup of subjects who did not take antihypertensive agents, both systolic and diastolic BP at the premotor stage were higher in female PD patients than in the healthy controls (Supplemental Tables 2, 3, Supplemental Fig. 1). Similarly, the serum levels of T-Cho and LDL-Cho showed a premotor decline in the male statin-free subjects with PD, as observed in the analysis of the total population (Supplemental Tables 4, 5 and Supplemental Fig. 2).

Given that previous reports have suggested that low haemoglobin $(\mathrm{Hb})$ and uric acid (UA) levels are potential risk factors of $\mathrm{PD}^{20,21,23}$, we also investigated longitudinal changes in these factors. Both $\mathrm{Hb}$ and UA values decreased after the onset of motor symptoms in the male PD subjects, but a change at the premotor stage was not observed (Supplemental Fig. 3A,B). There were no detectable longitudinal differences in Hb or UA between the female PD and healthy controls (Supplemental Fig. 3A,B).

\section{Discussion}

In this study, our analysis using a linear mixed model detected an increase in BP in the premotor phase in the female PD patients. We also found that Ht decreased before motor symptoms onset in male PD patients. Furthermore, there was a premotor decline in serum T-Cho and LDL-Cho levels in the male patients, whereas these values decreased after motor symptoms onset in the female patients.

Additionally, the systolic and diastolic BP values in the female PD subjects were high but within normal range before motor symptoms onset, but there was no difference in the systolic and diastolic BP values in the males. Previous reports have suggested that systolic BP was negatively associated with PD risk in both sexes ${ }^{24}$. Other studies have reported that high BP in the normal range was associated with PD in women, suggesting a possible influence of oestrogen and/or autonomic dysfunction ${ }^{25,26}$. Given that oestrogen has been implicated in 


\begin{tabular}{|c|c|c|c|c|}
\hline \multicolumn{5}{|l|}{ Male } \\
\hline Height & $167.0 \pm 7.0(153.1-180.9)$ & $167.8 \pm 6.1(155.8-179.7)$ & 99.5 & 0.614 \\
\hline Weight & $63.6 \pm 7.0(45.9-81.3)$ & $67.0 \pm 7.9(51.5-82.6)$ & 94.9 & 0.094 \\
\hline BMI & $22.7 \pm 1.9(19.0-26.4)$ & $23.9 \pm 2.4(19.2-28.7)$ & 94.7 & 0.026 \\
\hline Systolic BP & $126.1 \pm 13.1(100.3-151.9)$ & $123.5 \pm 11.4(101.0-146.0)$ & 102.1 & 0.382 \\
\hline Diastolic BP & $77.6 \pm 6.8(64.1-91.0)$ & $78.1 \pm 6.9(64.6-91.7)$ & 99.3 & 0.739 \\
\hline WBC & $5374.9 \pm 943.4(3509.2-7240.7)$ & $5449.3 \pm 1386.8(2718.0-8180.6)$ & 98.6 & 0.817 \\
\hline $\mathrm{RBC}$ & $461.2 \pm 41.2(380.0-542.5)$ & $470.7 \pm 32.2(407.3-534.2)$ & 98.0 & 0.276 \\
\hline $\mathrm{Hb}$ & $14.5 \pm 1.0(12.5-16.5)$ & $14.7 \pm 0.9(13.0-16.5)$ & 93.4 & 0.295 \\
\hline $\mathrm{Ht}$ & $42.2 \pm 3.0(36.3-48.0)$ & $44.0 \pm 2.6(38.9-49.1)$ & 98.4 & 0.008 \\
\hline Plt & $20.4 \pm 3.8(13.0-27.9)$ & $21.9 \pm 5.4(11.2-32.5)$ & 95.9 & 0.249 \\
\hline AST & $22.8 \pm 4.0(14.9-30.8)$ & $22.6 \pm 5.0(12.7-32.4)$ & 101.2 & 0.827 \\
\hline ALT & $21.7 \pm 8.2(5.5-37.8)$ & $22.9 \pm 9.0(5.2-40.7)$ & 94.4 & 0.560 \\
\hline$\gamma$-GTP & $44.1 \pm 33.1(-22.0-110.2)$ & $38.4 \pm 19.2(0.9-76.3)$ & 114.1 & 0.359 \\
\hline T-Cho & $190.7 \pm 33.2(124.9-256.5)$ & $212.4 \pm 21.9(169.3-255.5)$ & 89.8 & 0.001 \\
\hline TG & $108.2 \pm 65.7(-21.3-237.7)$ & $128.3 \pm 49.1(31.5-225.0)$ & 84.4 & 0.138 \\
\hline LDL-Cho & $107.8 \pm 25.9(56.8-158.8)$ & $122.9 \pm 24.2(75.4-170.5)$ & 87.7 & 0.015 \\
\hline HDL-Cho & $57.1 \pm 11.2(35.1-79.1)$ & $59.7 \pm 11.5(37.0-82.4)$ & 95.6 & 0.360 \\
\hline HbAlc & $5.6 \pm 0.6(4.5-6.8)$ & $5.8 \pm 0.3(5.2-6.3)$ & 97.4 & 0.125 \\
\hline $\mathrm{Cr}$ & $0.8 \pm 0.1(0.6-1.1)$ & $0.9 \pm 0.1(0.7-1.1)$ & 91.5 & 0.012 \\
\hline UA & $5.6 \pm 1.3(3.0-8.1)$ & $6.0 \pm 0.8(4.4-7.7)$ & 92.4 & 0.063 \\
\hline $\mathrm{TP}$ & $7.3 \pm 0.4(6.6-8.0)$ & $7.2 \pm 0.3(6.6-7.8)$ & 101.5 & 0.185 \\
\hline \multicolumn{5}{|l|}{ Female } \\
\hline Height & $151.9 \pm 5.0(142.1-161.7)$ & $154.8 \pm 6.1(142.8-166.8)$ & 98.1 & 0.045 \\
\hline Weight & $49.8 \pm 9.1(31.9-67.8)$ & $51.6 \pm 7.0(37.8-65.5)$ & 96.5 & 0.344 \\
\hline BMI & $22.1 \pm 4.0(14.2-30.1)$ & $21.6 \pm 3.2(15.3-27.9)$ & 102.3 & 0.560 \\
\hline Systolic BP & $126.6 \pm 12.1(102.6-150.5)$ & $119.7 \pm 13.4(93.3-146.1)$ & 105.8 & 0.035 \\
\hline Diastolic BP & $75.0 \pm 7.35(61.2-90.2)$ & $71.4 \pm 8.8(53.9-88.8)$ & 106.1 & 0.042 \\
\hline WBC & $4931.1 \pm 1103.5(2738.6-7123.7)$ & $4566.5 \pm 1014.3(2567.8-6565.3)$ & 108.0 & 0.161 \\
\hline $\mathrm{RBC}$ & $430.2 \pm 31.0(368.8-491.7)$ & $437.3 \pm 26.9(384.3-490.2)$ & 98.4 & 0.317 \\
\hline $\mathrm{Hb}$ & $13.3 \pm 0.9(11.5-15.1)$ & $13.5 \pm 0.7(12.2-14.8)$ & 98.4 & 0.237 \\
\hline $\mathrm{Ht}$ & $39.7 \pm 2.8(34.2-45.2)$ & $40.7 \pm 1.9(37.0-44.4)$ & 97.6 & 0.066 \\
\hline Plt & $22.2 \pm 4.9(12.3-32.1)$ & $22.2 \pm 3.9(14.6-29.9)$ & 99.8 & 0.971 \\
\hline AST & $23.6 \pm 4.7(14.3-32.9)$ & $21.4 \pm 3.4(14.6-28.1)$ & 110.4 & 0.020 \\
\hline ALT & $19.1 \pm 5.2(8.9-29.4)$ & $19.2 \pm 5.3(8.7-29.6)$ & 99.8 & 0.981 \\
\hline$\gamma$-GTP & $26.6 \pm 17.4(-7.7-60.9)$ & $24.5 \pm 10.9(3.1-46.0)$ & 108.5 & 0.518 \\
\hline T-Cho & $216.2 \pm 26.9(162.8-269.5)$ & $234.9 \pm 30.6(174.7-295.1)$ & 92.0 & 0.012 \\
\hline TG & $94.8 \pm 25.0(45.5-144.1)$ & $92.8 \pm 24.0(45.5-140.1)$ & 102.2 & 0.736 \\
\hline LDL-Cho & $122.6 \pm 25.3(72.6-172.7)$ & $132.1 \pm 25.0(83.0-181.2)$ & 92.8 & 0.131 \\
\hline HDL-Cho & $68.3 \pm 15.0(38.6-97.9)$ & $74.3 \pm 15.8(43.2-105.3)$ & 91.9 & 0.124 \\
\hline HbAlc & $5.7 \pm 0.2(5.2-6.2)$ & $5.8 \pm 0.3(5.2-6.3)$ & 99.2 & 0.491 \\
\hline $\mathrm{Cr}$ & $0.6 \pm 0.1(0.5-0.8)$ & $0.6 \pm 0.1(0.5-0.8)$ & 100.4 & 0.883 \\
\hline UA & $4.4 \pm 0.9(2.5-6.2)$ & $4.6 \pm 0.9(2.9-6.3)$ & 95.2 & 0.323 \\
\hline $\mathrm{TP}$ & $7.2 \pm 0.4(6.3-8.0)$ & $7.2 \pm 0.3(6.5-7.8)$ & 100.0 & 0.972 \\
\hline
\end{tabular}

Table 2. Comparison of baseline data between PD patients and healthy controls. Data represent the mean \pm standard error. $P D$ Parkinson's disease, $H C$ healthy control, $B M I$ body mass index, $B P$ blood pressure, $W B C$ white blood cell count, $R B C$ red blood cell count, $H b$ haemoglobin, $H t$ haematocrit, $P l t$ platelet count, $A L T$ alanine aminotransferase, AST aspartate aminotransferase, $\gamma$-GTP gamma-glutamyl transpeptidase, $T$-Cho total cholesterol, $T G$ triglyceride, $L D L$-Cho low-density lipoprotein cholesterol, $H D L$-Cho high-density lipoprotein cholesterol, $\mathrm{HbA1c}$ haemoglobin A1c, $\mathrm{Cr}$ creatinine, $U A$ uric acid, $T P$ total protein.

both hypertension ${ }^{27,28}$ and PD pathogenesis ${ }^{29,30}$, hormonal factors may underlie the sex-specific alteration of BP in the premotor phase of PD.

Our study showed that $\mathrm{Ht}$ values decreased before the motor symptoms onset in male PD patients, but $\mathrm{Hb}$ values showed no change. The biological indices related to anaemia have previously been shown to be risk factors of PD in various reports ${ }^{23}$. For example, a population-based cohort study showed that subjects with anaemia, especially with iron deficiency, were more likely to develop PD than were non-anaemic subjects ${ }^{31}$. The serum 
Male
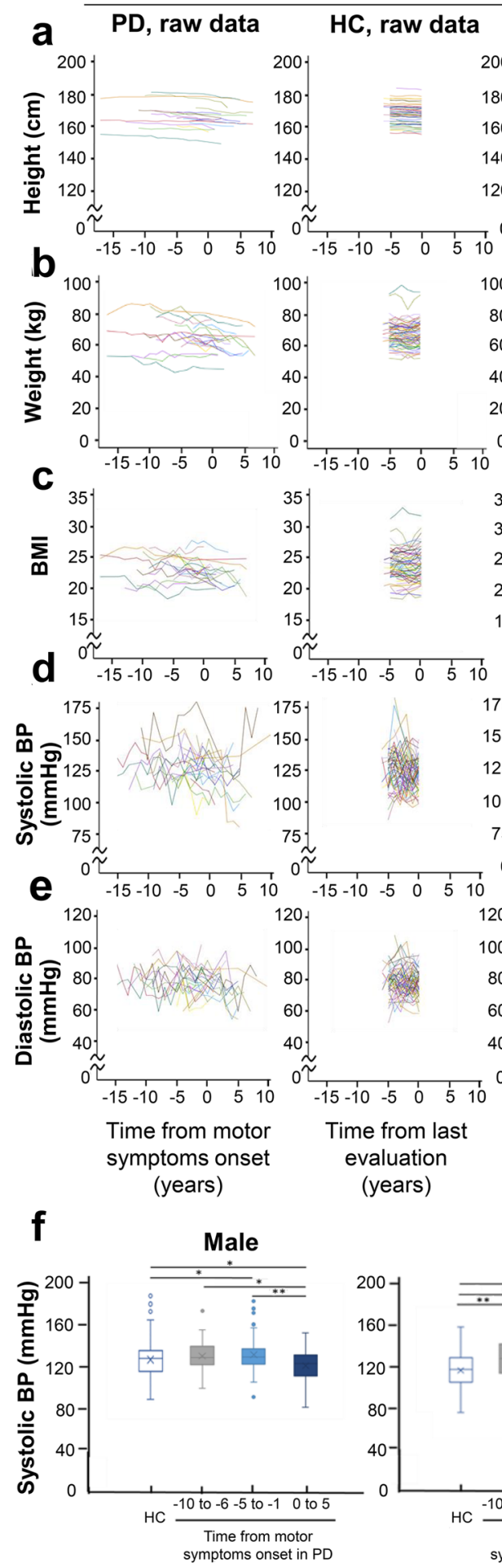
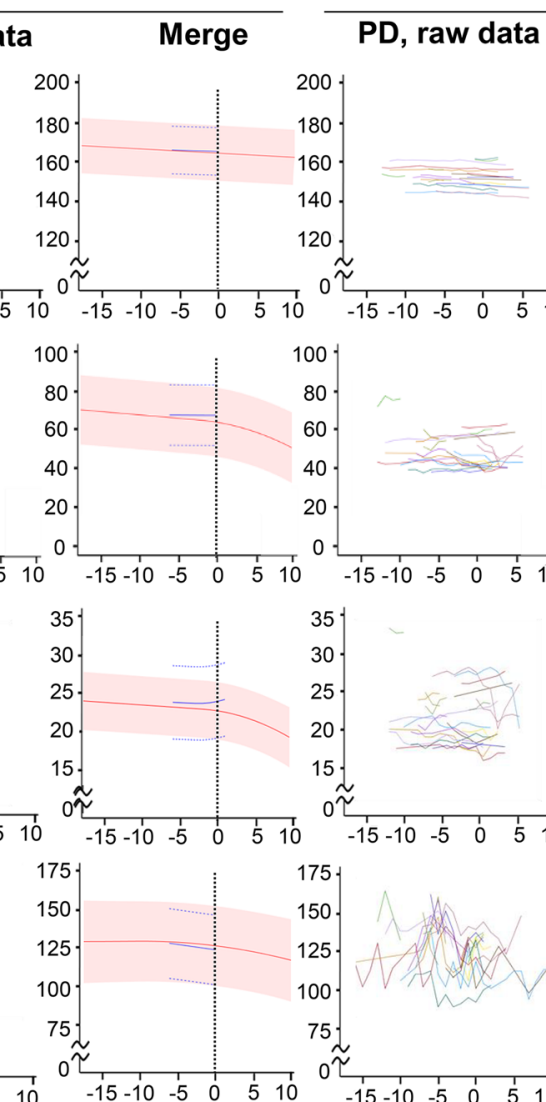

$\mathrm{PD}$, raw data $\mathrm{HC}$, raw data

Female
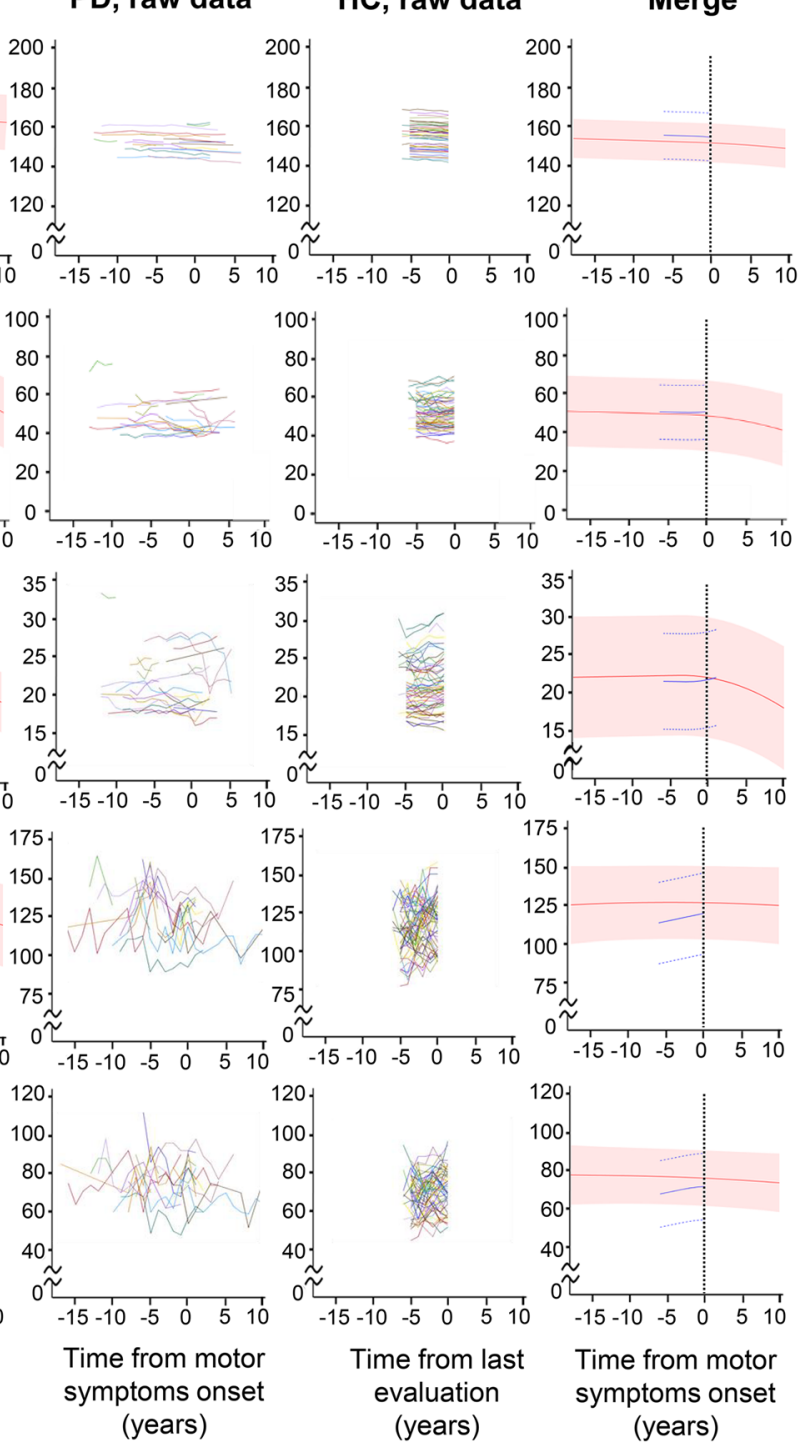

Figure 2. Longitudinal changes in anthropometric markers. (a-e) Raw data and estimated average trajectories of anthropometric and physical indices. Raw data for height (a), weight (b), BMI (c), systolic BP (d) and diastolic BP (e) are plotted against years relative to the onset of motor symptoms in patients with $P D(n=45$ : male, $n=22$; female, $n=23$ ) or years from the last evaluation in healthy controls $(n=120:$ male, $n=60$; female, $\mathrm{n}=60$ ). In raw data graphs, each polygonal line represents longitudinal data from a single participant. The estimated average trajectory and its $95 \%$ confidence intervals estimated with a linear mixed model are plotted for each marker in patients with PD (red line and shaded area, respectively) and healthy controls (blue lines) in the merged graphs. (f,g) Box plot of anthropometric and physical indices. Values of systolic BP (f), diastolic BP (g) in each clinical stage per 5 years of PD and in HCs are shown in a plot box. ${ }^{\star} p<0.05$ and ${ }^{* *} p<0.001$. Turkey multiple comparison tests $(\mathbf{f}, \mathbf{g})$. The horizontal line in the box plot indicates the median, and the cross mark indicates the average. $P D$ Parkinson's disease, $H C$ healthy control, $B M I$ body mass index, $B P$ blood pressure. 
Male
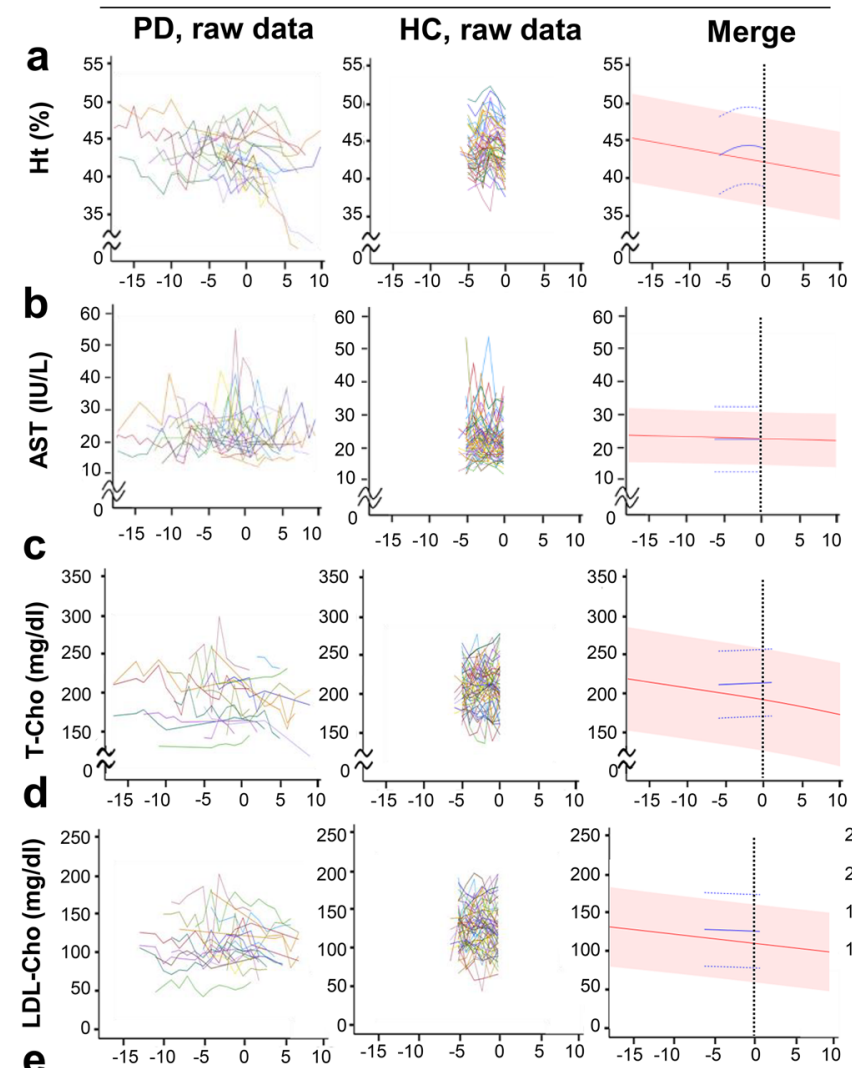

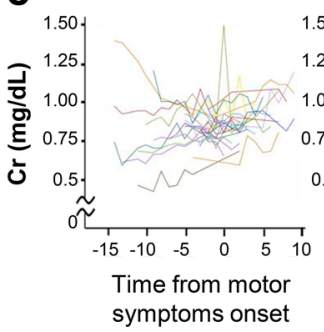

(years)

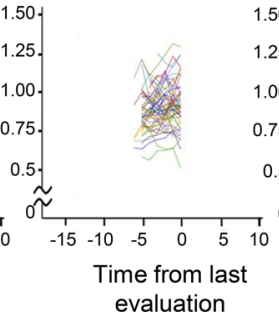

(years)

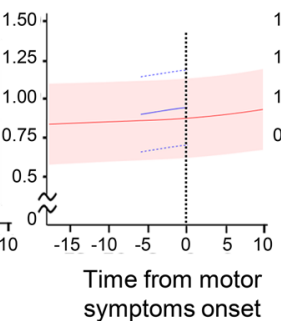

(years)

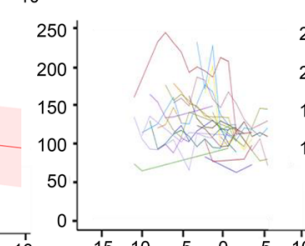

Female
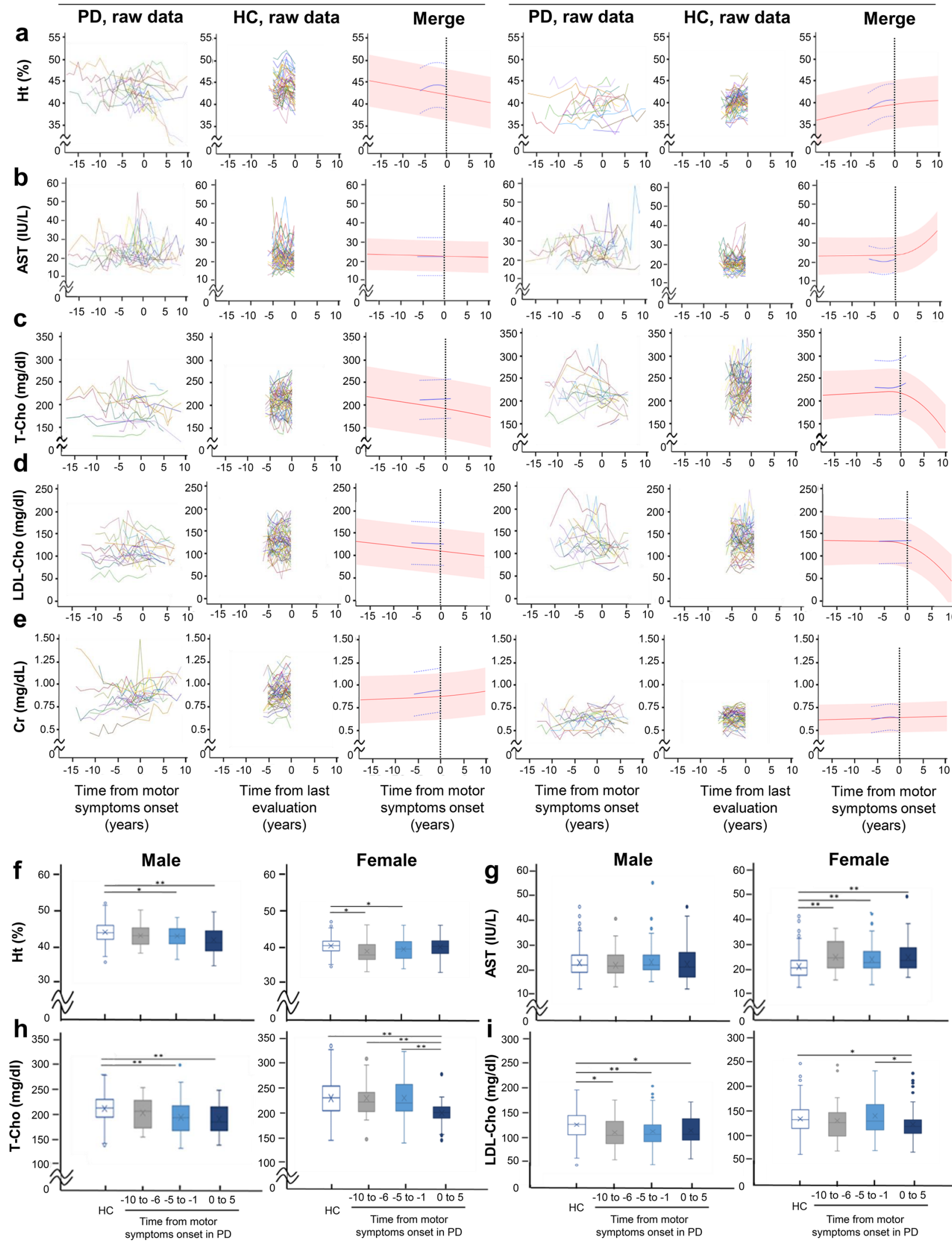

(years)
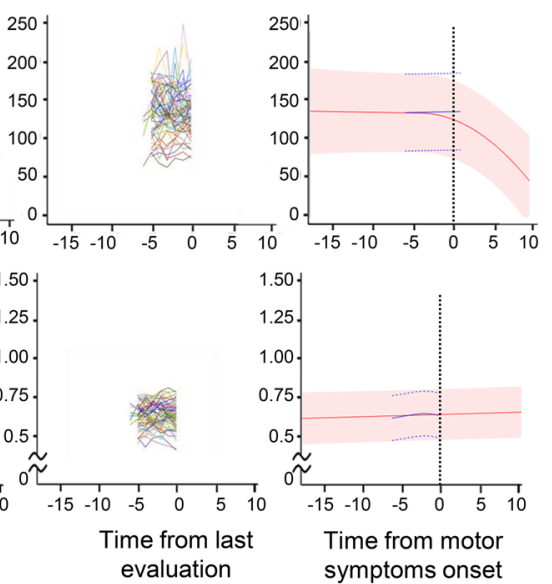

(years)

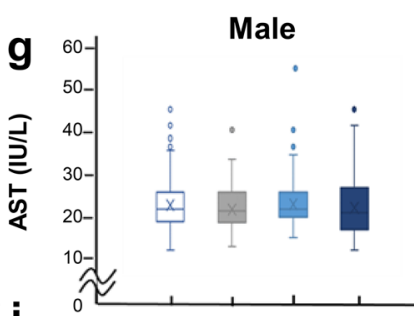

(years)

Female

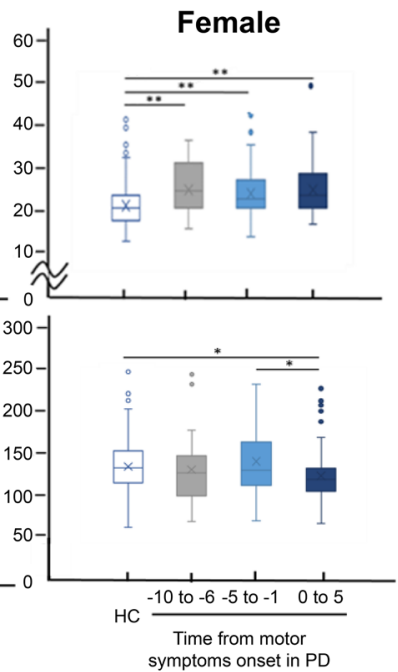


«Figure 3. Longitudinal changes in serological markers. (a-e) Raw data and estimated average trajectories of serological indices. Raw data for Ht (a), AST (b), T-Cho (c), LDL-Cho (d) and Cr (e) are plotted against years relative to the onset of motor symptoms in patients with PD $(n=45$ : male, $n=22$; female, $n=23)$ or years from the last evaluation in healthy controls $(n=120$ : male, $n=60$; female, $n=60)$. In raw data graphs, each polygonal line represents longitudinal data from a single participant. The estimated average trajectory and its 95\% confidence intervals estimated with a linear mixed model are plotted for each marker in patients with PD (red line and shaded area, respectively) and healthy controls (blue lines) in the merged graphs. (f-i) Box plot of serological indices. Values of Ht (f), AST (g), T-Cho (h) and LDL-Cho (i) in each clinical stage per 5 years of $\mathrm{PD}$ and in HCs are shown in a plot box. ${ }^{*} p<0.05$ and ${ }^{* *} p<0.001$. Turkey multiple comparison tests $(\mathbf{f}-\mathbf{i})$. The horizontal line in the box plot indicates the median, and the cross mark in the box plot indicates the average. PD Parkinson's disease, HC healthy control, HT haematocrit, AST aspartate aminotransferase, T-Cho total cholesterol, $L D L$-Cho low-density lipoprotein cholesterol, $\mathrm{Cr}$ creatinine.

Ht level reflects the volume of red blood cells relative to the total blood volume ${ }^{32}$. Given that subjects with nonanaemic iron deficiency have normal $\mathrm{Hb}$ and low $\mathrm{Ht}$ and ferritin levels $\mathrm{s}^{33}$, our results indicated dysregulation of iron metabolism in premotor PD individuals. It is well-known that nigral iron content is increased in $\mathrm{PD}^{34}$. Iron deposition in the nigra occurs even in the preclinical phase of LRRK2 and PRKN mutation carriers ${ }^{35}$. Serum ferritin is higher in postmenopausal women than in premenopausal females ${ }^{36}$, and the majority of the female $\mathrm{PD}$ patients in our study were near the menopausal age. This finding might reflect sex-specific alteration of $\mathrm{Ht}$ in our cohort.

Our analysis also showed that T-Cho and LDL-Cho started to decrease before the onset of motor symptoms in the males and after onset in the females. Previous reports have stated that higher levels of T-Cho or LDL-Cho were associated with a decreased risk of developing PD, particularly in males ${ }^{18}$. In support of this observation, our previous study demonstrated that T-Cho levels decreased in male, but not female, at-risk PD subjects who had multiple prodromal symptoms but no motor/cognitive deficits ${ }^{37}$. Dysregulation of Cho could affect the nervous system in multiple ways, and Cho dysregulation has been reported in a variety of neurodegenerative diseases ${ }^{38}$. Lipid metabolism abnormalities have been considered a causative factor of $\mathrm{PD}^{39}$, and high intake of dietary Cho is likely protective against $\mathrm{PD}$ pathogenesis ${ }^{40}$. Although the mechanism underlying the association between Cho and $\mathrm{PD}$ is not yet known, intestinal absorption of Cho is possibly inhibited by gastrointestinal dysfunction caused by $\alpha$-synuclein deposition before motor symptoms onset ${ }^{41}$. Accelerated lipoprotein metabolism due to oestrogen or apolipoprotein E phenotype is potentially attributable to sex-specific alteration in Cho in pre-motor $\mathrm{PD}^{42,43}$.

Our study had several limitations. This was a retrospective study that only included PD patients who had past health examination data before motor symptoms onset and who consented to submit their data for this study. Second, our sample size was small and limited by region and race, and items that were not included in routine health check-ups were not analysed. Third, since we selected the best fit model from among the several candidate models, we could not exclude the influence of multiplicity on our results. Consequently, we could not exclude selection bias completely, but analysing health examination data to investigate the changes in biological markers before motor symptoms onset could be generally applied to preclinical studies of other neurodegenerative analyses. Prospective studies with a larger sample size should be conducted in the future.

\section{Conclusions}

We found that BP, Ht and serum Cho levels were potential premotor biomarkers of PD. The changes in anthropometric and serological indices before motor symptoms onset in PD subjects were different between males and females.

\section{Materials and methods}

Ethics. This study was conducted in accordance with the Declaration of Helsinki, the Ethics Guidelines for Human Genome/Gene Analysis Research and the Ethical Guidelines for Medical and Health Research Involving Human Subjects endorsed by the Japanese government. The study protocol was approved by the Ethics Review Committee of Nagoya University Graduate School of Medicine. We obtained written informed consent from all participants before inclusion in this study.

Participants. PD patients were recruited from Kumiai Kosei Hospital and Nagoya University Hospital. The principal inclusion criteria were those of the UK Parkinson's Disease Society Brain Bank clinical diagnostic criteria $^{44}$ and either reduction uptake in ${ }^{123}$ I-metaiodobenzylguanidine cardiac scintigraphy or hyposmia confirmed by the odour stick identification test for Japanese ${ }^{45}$. We excluded PD patients for whom health checkup data before motor symptoms onset were not available, those who had a family history of PD and those who had a neurodegenerative disease other than PD.

We also recruited healthy controls from health checkup centres at Daido Hospital; they had all submitted health checkup data for $>4$ years from the last evaluation and provided consent. To compare the results of the health checkup data of the controls with those of the PD patients who had not noticed motor symptoms, we matched the last evaluation age of the healthy controls with the age of motor symptoms onset of the PD patients. We excluded subjects with PD, with neurodegenerative disease other than PD, with a family history of PD, with serious complications and with prodromal symptoms of PD from the healthy controls. We used the self-reported questionnaires for exploring prodromal symptoms of PD in health checkup examinees ${ }^{37}$. 
Data acquisition. We acquired and assessed longitudinal data from periodic health check-ups for all patients and controls. In Japan, the law requires that employees must get a health examination that includes biochemical and anthropometric examinations once every year. Moreover, some non-employees spontaneously undergo health check-ups every year, which is why some people have their own longitudinal data either at hospitals or their homes.

The following biochemical parameters are measured commonly at cooperating facilities: height, body weight, BMI, systolic BP, diastolic BP, white blood cell count, Hb, Ht, platelet count, AST, alanine aminotransferase, gamma-glutamyl transpeptidase, T-Cho, triglycerides, LDL-Cho, high-density lipoprotein cholesterol, haemoglobin A1c (HbA1c), Cr, UA and total protein. The HbA1c level was estimated as the National Glycohaemoglobin Standardisation Programme-equivalent value (percent). BMI was calculated from the obtained height and body weight values.

In patients with $\mathrm{PD}$, we analysed data obtained both before diagnosis and during follow-up after diagnosis. In the healthy controls, we analysed data obtained before the last evaluation. We evaluated all data by determining the differences in the clinical features of PD between males and females ${ }^{46,47}$.

Statistical analysis. We analysed all data for each sex separately. By using a mixed-effects model, including random intercepts, we estimated average trajectories for each biomarker across years ${ }^{48}$. The model included polynomial basis functions to incorporate quadratic smoothing. Estimated values and 95\% confidence intervals were computed from -17 to +13 years relative to symptom onset at 1 -year intervals in the patients with PD. For each index, we selected the best fit model among the several candidate models based on the goodness of fit evaluation. Multiple comparisons using statistical testing were not performed when selecting the best fitted model. Student's $t$-test (or the Mann-Whitney $U$-test) was performed to compare the distribution of continuous variable between the two groups. The chi-squared test was used to compare the distribution of categorical variables between the two groups. Pearson correlation coefficients were used to analyse correlations among parameters. Continuous and categorical data are presented as the mean \pm SD and frequency (proportion), respectively, unless stated otherwise. We considered $p<0.05$ to be indicative of statistical significance. The Statistical Package for the Social Sciences 25.0 J software (IBM Japan, Tokyo, Japan) and SAS version 9.4 (SAS Institute, Inc., Cary, NC, USA) were used to perform all statistical analyses.

\section{Data availability}

The datasets generated during and/or analysed during the current study are available from the corresponding author on reasonable request.

Received: 27 March 2020; Accepted: 10 November 2020

Published online: 25 November 2020

\section{References}

1. de Lau, L. M. \& Breteler, M. M. Epidemiology of Parkinson's disease. Lancet Neurol. 5, 525-535 (2006).

2. Schapira, A. H. V., Olanow, C. W., Greenamyre, J. T. \& Bezard, E. Slowing of neurodegeneration in Parkinson's disease and Huntington's disease: Future therapeutic perspectives. Lancet 384, 545-555 (2014).

3. Parkinson, J. An essay on the Shaking Palsy. Arch. Neurol. 20, 441-445 (1969).

4. Simuni, T. \& Sethi, K. Nonmotor manifestations of Parkinson's disease. Ann. Neurol. 64, 65-80 (2008).

5. Kalia, L. V. \& Lang, A. E. Parkinson's disease. Lancet 386, 896-912 (2015).

6. Postuma, R. B. \& Berg, D. Advances in markers of prodromal Parkinson disease. Nat. Rev. Neurol. 12, 622-634 (2016).

7. Kordower, J. H. et al. Disease duration and the integrity of the nigrostriatal system in Parkinson's disease. Brain 136, 2419-2431 (2013).

8. Weydt, P. et al. Neurofilament levels as biomarkers in asymptomatic and symptomatic familial amyotrophic lateral sclerosis. Ann. Neurol. 79, 152-158 (2016).

9. van der Ende, E. L. et al. Serum neurofilament light chain in genetic frontotemporal dementia: A longitudinal, multicentre cohort study. Lancet Neurol. 18, 1103-1111 (2019).

10. Preische, O. et al. Serum neurofilament dynamics predicts neurodegeneration and clinical progression in presymptomatic Alzheimer's disease. Nat. Med. 25, 277-283 (2019).

11. Jack, C. R. et al. Tracking pathophysiological processes in Alzheimer's disease: An updated hypothetical model of dynamic biomarkers. Lancet Neurol. 12, 207-216 (2013).

12. Gendron, T. F. et al. Poly(GP) proteins are a useful pharmacodynamic marker for C9ORF72-associated amyotrophic lateral sclerosis. Sci. Transl. Med. 9, 7866 (2017).

13. Jacobi, H. et al. Biological and clinical characteristics of individuals at risk for spinocerebellar ataxia types 1, 2, 3, and 6 in the longitudinal RISCA study: Analysis of baseline data. Lancet Neurol. 12, 650-658 (2013).

14. Tabrizi, S. J. et al. Biological and clinical changes in premanifest and early stage Huntington's disease in the TRACK-HD study: The 12-month longitudinal analysis. Lancet Neurol. 10, 31-42 (2011).

15. Ragonese, P. et al. Height as a potential indicator of early life events predicting parkinson's disease: A case-control study. Mov. Disord. 22, 2263-2267 (2007).

16. Chen, H., Zhang, S. M., Herna, M. A., Willett, W. C. \& Ascherio, A. Weight loss in Parkinson's disease. Ann. Neurol. 2, 676-679 (2003).

17. Kasten, M., Tadic, V. \& Klein, C. Anemia or low hemoglobin levels prededing PD: A case-control study. Neurology 2, 1655-1657 (2010).

18. Rozani, V. et al. Higher serum cholesterol and decreased Parkinson's disease risk: A statin-free cohort study. Mov. Disord. 33, 1298-1305 (2018).

19. Huang, X., Abbott, R. D., Petrovitch, H., Mailman, R. B. \& Ross, G. W. Low LDL cholesterol and increased risk of Parkinson's disease: Prospective results from Honolulu-Asia aging study. Mov. Disord. 23, 1013-1018 (2008).

20. de Lau, L. M. L., Koudstaal, P. J., Hofman, A. \& Breteler, M. M. B. Serum uric acid levels and the risk of Parkinson disease. Ann. Neurol. 58, 797-800 (2005). 
21. Gao, X., O'Reilly, E. J., Schwarzschild, M. A. \& Ascherio, A. Prospective study of plasma urate and risk of Parkinson disease in men and women. Neurology 86, 520-526 (2016).

22. Hijikata, Y. et al. Biomarker-based analysis of preclinical progression in spinal and bulbar muscular atrophy. Neurology 90, E1501E1509 (2018).

23. Savica, R. et al. Anemia or low hemoglobin levels preceding Parkinson disease: A case-control study. Neurology 73, 1381-1387 (2009).

24. Vikdahl, M., Bäckman, L., Johansson, I., Forsgren, L. \& Haglin, L. Cardiovascular risk factors and the risk of Parkinson's disease. Eur. J. Clin. Nutr. 69, 729-733 (2015).

25. Qiu, C. et al. Association of blood pressure and hypertension with the risk of Parkinson disease: The national FINRISK study. Hypertension 57, 1094-1100 (2011).

26. Launer, L. J. et al. Lowering mid-life levels of systolic blood pressure as a public health strategy to reduce late-life dementia. Hypertension 38, 469-485 (2006).

27. Dgbrowska, B., Dgbrowski, A. \& Skrobowski, A. Parasympathetic withdrawal precedes spontaneous blood pressure elevations in women with primary hypertension?. Cardiology 87, 119-124 (1996).

28. Liu, S.-H. et al. The impact of estrogen supplementation to autonomic and sleep modulations in free-moving spontaneously hypertensive rats. Int. Heart J. 61, 128-137 (2020).

29. Hatano, T., Saiki, S., Okuzumi, A., Mohney, R. P. \& Hattori, N. Identification of novel biomarkers for Parkinson's disease by metabolomic technologies. J. Neurol. Neurosurg. Psychiatry 87, 295-301 (2016).

30. Gillies, G. E. \& McArthur, S. Independent influences of sex steroids of systemic and central origin in a rat model of Parkinson's disease: A contribution to sex-specific neuroprotection by estrogens. Horm. Behav. 57, 23-34 (2010).

31. Hong, C. T. et al. Newly diagnosed anemia increases risk of Parkinson's disease: A population-based cohort study. Sci. Rep. 6, 1-7 (2016).

32. Billett, H. H. Hemoglobin and Hematocrit. Clinical Methods: The History, Physical, and Laboratory Examinations 3rd edn (eds Walker, H. K. et al.) Ch. 151 (Butterworths, Boston, 1990).

33. Clénin, G. E. The treatment of iron deficiency without anaemia (in otherwise healthy persons). Swiss Med. Wkly. 147, 1-17 (2017).

34. Dexter, D. T. et al. Increased nigral iron content and alterations in other metal ions occurring in brain in Parkinson's disease. J. Neurochem. 52, 1830-1836 (1989).

35. Pyatigorskaya, N. et al. High nigral iron deposition in LRRK2 and Parkin mutation carriers using R2 ${ }^{\star}$ relaxometry. Mov. Disord. 30, 1077-1084 (2015).

36. Milman, N., Rosdahl, N., Lyhne, N., Jørgensen, T. \& Graudal, N. Iron status in Danish women aged 35-65 years: Relation to menstruation and method of contraception. Acta Obstet. Gynecol. Scand. 72, 601-605 (1993).

37. Hattori, M. et al. Subjects at risk of Parkinson's disease in health checkup examinees: Cross-sectional analysis of baseline data of the NaT-PROBE study. J. Neurol. https://doi.org/10.1007/s00415-020-09714-6 (2020).

38. Katsuno, M., Adachi, H. \& Sobue, G. The case for cholesterol. Nat. Med. 15, 253-254 (2009).

39. Galvagnion, C. The role of lipids interacting with $\alpha$-synuclein in the pathogenesis of Parkinson's disease. J. Parkinsons. Dis. 7, 433-450 (2017).

40. De Lau, L. M. L. et al. Dietary fatty acids and the risk of Parkinson disease: The Rotterdam Study. Neurology 64, 2040-2045 (2005).

41. Fasano, A., Visanji, N. P., Liu, L. W. C., Lang, A. E. \& Pfeiffer, R. F. Gastrointestinal dysfunction in Parkinson's disease. Lancet Neurol. 14, 625-639 (2015).

42. Knopp, R. H. et al. Sex differences in lipoprotein metabolism an dietary response: Basis in hormonal differences and implications for cardiovascular disease. Curr. Cardiol. Rep. 8, 452-459 (2006).

43. Schaefer, E. J. et al. Effects of gender and menopausal status on the association of apolipoprotein E phenotype with plasma lipoprotein levels: Results from the Framingham Offspring Study. Arterioscler. Thromb. 14, 1105-1113 (1994).

44. Hughes, A. J., Daniel, S. E., Kilford, L. \& Lees, A. J. Accuracy of clinical diagnosis of idiopathic Parkinson's disease: A clinicopathological study of 100 cases. J. Neurol. Neurosurg. Psychiatry 55, 181-184 (1992).

45. Saito, S. et al. Development of a smell identification test using a novel stick-type odor presentation kit. Chem. Senses 31, 379-391 (2006).

46. Jurado-Coronel, J. C. et al. Sex differences in Parkinson's disease: Features on clinical symptoms, treatment outcome, sexual hormones and genetics. Front. Neuroendocrinol. 50, 18-30 (2018).

47. Smith, K. M. \& Dahodwala, N. Sex differences in Parkinson's disease and other movement disorders. Exp. Neurol. 259, 44-56 (2014).

48. Yau, W. Y. W. et al. Longitudinal assessment of neuroimaging and clinical markers in autosomal dominant Alzheimer's disease: A prospective cohort study. Lancet Neurol. 14, 804-813 (2015).

\section{Acknowledgements}

The authors would like to thank Enago (www.enago.jp) for the English language review.

\section{Author contributions}

K.Y., M.H., H.W. and Ma.K. conceived and designed the study. K.Y., M.H., Y.S., Y.T., M.S., A.Ho. and Mo.K. contributed to the acquisition of clinical data. K.Y., A.Ha and Ma.K. performed analysis and interpretation of the data. A.Hi. performed statistical analysis. K.Y. drafted the manuscript and Ma.K. revised it for intellectual content.

\section{Funding}

This work was funded by The Research Funding for Longevity Sciences (Nos. 28-8 and 19-20) from the National Centre for Geriatrics and Gerontology (NCGG), Japan and grants from Japan Agency for Medical Research and Development (Nos. 19dk0207027h0004, 19lk0201101h0001, and 19dm0107155h0001).

\section{Competing interests}

Drs. Yokoi, Hattori, Satake, Tanaka, Sato, Hashizume, Hori, Kawashima and Hirakawa report no disclosures. Dr. Watanabe is supported by Otsuka Pharmaceutical Co. Ltd., Kyowa Hakko Kirin Co. Ltd., Takeda Pharmaceutical Co. Ltd., Dainippon Sumitomo Pharma Co. Ltd., FP Pharmaceutical Corporation, Eisai Co. Ltd., Daiichi Sankyo Company Limited and Novartis Pharma K.K., and grants from Otsuka Pharmaceutical Co. Ltd. and FUJIFILM RI Pharma Co. Ltd. Dr. Katsuno is supported by a JSPS KAKENHI Grant Number JP17H04195, grants from the Japan Agency for Medical Research and Development (Nos. 19ek0109221h0003, 19ek0109359h0002, $19 \mathrm{dk} 0207027 \mathrm{~h} 0004,19 \mathrm{k} 0201101 \mathrm{~h} 0001$ and 19dm0107155h0001), a grant from the Naito Foundation and a grant from the Hori Sciences and Arts Foundation. He received honoraria from Takeda Pharmaceutical Co. Ltd., 
Alnylam Japan, Daiichi Sankyo Co. Ltd., Otsuka Pharmaceutical Co. Ltd., Novartis Pharma Co. Ltd., Biogen Japan and UCB Japan and grants from Zenyaku Kogyo Co. Ltd., Japan Blood Products Organization, MitsubishiTanabe Pharma, CSL Behring Co. Ltd., Dainippon Sumitomo Pharma Co. Ltd., Otsuka Pharmaceutical Co. Ltd. and Daiichi Sankyo Co. Ltd.

\section{Additional information}

Supplementary information is available for this paper at https://doi.org/10.1038/s41598-020-77415-1.

Correspondence and requests for materials should be addressed to M.K.

Reprints and permissions information is available at www.nature.com/reprints.

Publisher's note Springer Nature remains neutral with regard to jurisdictional claims in published maps and institutional affiliations.

Open Access This article is licensed under a Creative Commons Attribution 4.0 International License, which permits use, sharing, adaptation, distribution and reproduction in any medium or format, as long as you give appropriate credit to the original author(s) and the source, provide a link to the Creative Commons licence, and indicate if changes were made. The images or other third party material in this article are included in the article's Creative Commons licence, unless indicated otherwise in a credit line to the material. If material is not included in the article's Creative Commons licence and your intended use is not permitted by statutory regulation or exceeds the permitted use, you will need to obtain permission directly from the copyright holder. To view a copy of this licence, visit http://creativecommons.org/licenses/by/4.0/.

(C) The Author(s) 2020 